\title{
Enhancing value of quality assurance rounds in improving radiotherapy management: a retrospective analysis from King Hussein Cancer Center in Jordan
}

\author{
Jamal K. Khader, MD¹, Abdelatif M. Al-Mousa, MD, PhD¹, Issa A. Mohamad, MD¹, \\ Ramiz A. Abuhijlih, MD1', Sondos A. Al-Khatib, MD1', Anoud Z. Alnsour, MD1', Wafa A. Asha, MD1, \\ Shada W. Ramahi, PhD², Ali A. Hosni, MBBCh, PhD², Fawzi J. Abuhijla, MD, MSc ${ }^{1}$ \\ 1 Department of Radiation Oncology, King Hussein Cancer Center, Amman, Jordan; \\ ${ }^{2}$ Department of Biomedical Physics, King Faisal Specialist Hospital and Research Center, Riyadh, Saudi Arabia; \\ ${ }^{3}$ Department of Radiation Oncology, Princess Margaret Cancer Centre, Toronto, Canada
}

\begin{abstract}
Purpose: The quality assurance (QA) chart rounds are multidisciplinary meetings to review radiation therapy (RT) treatment plans. This study focus on describing the changes in RT management based on QA round reviews in a single institution.

Materials and Methods: After 9 full years of implementation, a retrospective review of all patients whose charts passed through departmental QA chart rounds from 2007 to 2015. The reviewed cases were presented for RT plan review; subcategorized based on decision in QA rounds into: approved, minor modifications or major modifications. Major modification defined as any substantial change which required patient re-simulation or re-planning prior to commencement of RT. Minor modification included treatment plan changes which didn't necessarily require RT re-planning. Results: Overall 7,149 RT treatment plans for different anatomical sites were reviewed at QA rounds. From these treatment plans, 6,654 (93\%) were approved, 144 (2\%) required minor modifications, while 351 (5\%) required major modifications. Major modification included changes in: selected RT dose $(96 / 351,27 \%)$, target volume definition $(127 / 351,36 \%)$, organs-at-risk contouring $(10 / 351,3 \%)$, dose volume objectives/constraints criteria $(90 / 351,26 \%)$, and intent of treatment $(28 / 351,8 \%)$. The RT plans which required major modification according to the tumor subtype were as follows: head and neck (104/904, 12\%), thoracic $(12 / 199,6 \%)$, gastrointestinal $(33 / 687,5 \%)$, skin $(5 / 106,5 \%)$, genitourinary $(16 / 359,4 \%)$, breast $(104 / 2387,4 \%)$, central nervous system $(36 / 846,4 \%)$, sarcoma (11/277, 4\%), pediatric $(7 / 251,3 \%)$, Iymphoma $(10 / 423,2 \%)$, gynecological tumors $(2 / 359,1 \%)$, and others $(11 / 351,3 \%)$.

Conclusion: Multi-disciplinary standardized QA chart rounds provide a comprehensive and an influential method on RT plans and/ or treatment decisions.
\end{abstract}

Keywords: Quality management, Multidisciplinary, Radiotherapy, Peer review

\section{Introduction}

Radiation therapy (RT) has always been an important treatment modality in cancer management. Advanced technologies within radiation oncology field have emerged globally through the past decades, which necessitates continuous quality assurance

Received 03 October 2018, Revised 21 January 2019, Accepted 20 February 2019.

Correspondence: Fawzi J. Abuhijla, MD, MSc, Department of Radiation Oncology, King Hussein Cancer Center, Queen Rania St., P.O. Box 1269, Amman 11941, Jordan. Tel: +962-796500577, Fax: +962-65342567, E-mail: fhijle@khcc.jo (http:// orcid.org/0000-0002-7264-6789)

(C) This is an Open Access article distributed under the terms of the Creative Commons Attribution Non-Commercial License (http://creativecommons.org/ licenses/by-nc/4.0/) which permits unrestricted non-commercial use, distribution, and reproduction in any medium, provided the original work is properly cited.

www.e-roj.org 
$(\mathrm{QA})$ to maintain adequate care and treatment delivery [1]. In particular, our region has seen a flux of new techniques in RT in recent years and while this has a positive impact on patient care [2-4], it also increases the concern of proper planning and chart $\mathrm{QA}$.

As per the World Health Organization, the definition for $\mathrm{QA}$ in RT is "all procedures that ensure consistency of the medical prescription, and safe fulfillment of that prescription, as regards to the dose to the target volume, together with minimal dose to normal tissue, minimal exposure to personnel and adequate patient monitoring aimed at determining the end result of the treatment" [5].

The $Q A$ rounds engage with the pre-treatment part of this definition, through checking for missing documentation in the patient chart [6] and reviewing RT plan details including prescribed dose and dose received by organs-at-risk (OAR) and target volumes. The variation in contouring the target volume is reported in literature [7], and the rounds would minimize this variation by reviewing the plans in order to achieve consensus agreement. Keeping in mind the differences in volume definitions related to the RT treatment modality used, due to higher precision needed through image-guided radiation therapy (IGRT), especially with more conformal and higher doses per fraction as in intensity-modulated radiotherapy (IMRT), stereotactic body radiotherapy (SBRT) [8]. It has been shown before that the more sophisticated the technique (as SBRT), the more need for OA [9].

Several reviews were published to assess the impact of QA rounds in RT from North America and Europe [10-12], as well as in Asia and Australia $[6,13]$. To our knowledge, this report is considered the first of its kind from RT departments in developing countries and the Middle East region. The work we present here is a retrospective analysis of the chart-care quality after 9 full years of implementation of the QA rounds. The data is stratified according to the modifications that were implemented after the $\mathrm{QA}$ rounds.

\section{Materials and Methods}

\section{Study participants}

A retrospective review was conducted for all cancer patients treated at our institution with radical and/or complex palliative external beam radiation therapy for whom the RT charts have been evaluated through departmental $\mathrm{QA}$ review rounds from January 2007 till December 2015. All primary tumor sites were included. Complex palliative cases included those who required $\mathrm{RT}$ to previously irradiated area or radical $\mathrm{RT}$ dose for metastatic disease.

Prior to RT plan presentation in QA rounds, it should be reviewed and approved by the treating radiation oncologist. The implementation of IMRT was introduced since 2010 and has been used mainly for head \& neck and prostate cancers. Patient-specific-QA for IMRT plans is done by the medical physicists after approval in $\mathrm{QA}$ rounds. The RT is not routinely delivered unless the plan is reviewed in the $Q A$ rounds and the review results are recorded in the pre-treatment check list in the patients' chart. After this stage of OA rounds, all approved plans are ready to go and start treatment. All plans were done using Pinnacle treatment planning system (Philips Medical Systems, Best, The Netherlands).

\section{Quality assurance review rounds}

The $Q A$ review rounds are held for 1 hour session 2-3 times per week on a regular basis except for holidays. The panel participants ideally includes all radiation oncologists (staff and trainees), senior medical physicst (including dosimetrists and the radiation safety officer), oncology nurses and senior radiation therapy technicians (RTT). However, as a minimum, the panel should include three staff radiation oncologists, one senior medical physicist, senior radiation dosimetrist, senior radiation therapist, the radiation protection officer, radiation oncology nurses and radiation oncology residents.

The list of patients for review is sent prior to the $\mathrm{QA}$ review round with a maximum of 15 patients per meeting and contains the patients' medical number, treating radiation oncologist, primary tumor site and RT details including dose, fractionation and radiation technique. The radiation oncologist presents a brief history and management plan, and the dosimetrist displays the radiotherapy treatment plan. The $\mathrm{OA}$ review team then proceeds to discuss each case and the plan is considered approved if the majority agree to the treatment management. Otherwise, further discussion is needed to agree on how the management should be modified.

\section{Modification}

As per departmental policy, major modifications are a cause to halt or postpone the start of the RT, whereas minor modifications will not affect the start of treatment. Major modifications are defined as any substantial change which requires patient re-simulation and/or re-planning. This include changes in dose fractionation (total dose, or dose per fraction), dose volume objectives/constraints which require re-planning, change in RT technique (conventional vs. modulated delivery), intent of treatment (curative vs. palliative) and target volume 
definition which would lead to changes in clinical target volume (CTV) or changes in conventional field design by more than $1 \mathrm{~cm}$ (e.g., increasing the upper field for breast tangents by more than $1 \mathrm{~cm}$ is considered a major modification).

Minor modifications defined as any plan change which doesn't necessarily require re-planning. This include minor changes in the planning target volume (PTV) that won't change the dosimetric planning outcome, re-evaluating the acceptance criteria for PTV and OAR doses to an extent that doesn't require re-planning merely changing the recorded value. Those minor changes while not affecting the current plan being considered but will be taken as points for improvement for future similar cases. Also, minor modifications include changes in multi-leaf collimator as a blocking (not a modulating) device or radiation field by less than $1 \mathrm{~cm}$ for conventional RT plans.

\section{Data collection}

All comments and decisions taken per case during the $\mathrm{OA}$ rounds are prospectively documented and maintained in the radiation oncology department database records. These comments include the pre-discussion list, debate at the $\mathrm{QA}$ rounds and the final decision (approved, minor modifications and major modifications).

\section{Results}

The database of QA rounds from beginning of January 2007 till the end of December 2015 was reviewed. Primary tumor sites were divided into 12 broad tumor categories: breast, central nervous system, gastrointestinal, gynecologic, genitourinary, head \&t neck, thoracic, lymphoma, sarcoma, skin, pediatric and others as unknown primary, leukemia and benign tumors.

Overall 7,149 RT treatment plans for different anatomical sites were reviewed at our departmental OA rounds as seen in Fig. 1. Table 1 breaks down the approved, major and minor modification per site, while Table 2 presents same data per each year. The rise seen in the number of modifications in 2010 coincides with the implementation of IMRT. The planning technique used for these plans were: two-dimensional which included two opposing plans, 3 or 4 field conventional plans as 1,707 , forward IMRT (F.IMRT) mainly for breast plans as 2,355, three-dimensional conformal radiotherapy (3D-CRT) as 2,286 while IMRT plan were 801. The distribution of different radiotherapy planning technique for each site is shown in Table 3.

Among the 7,149 RT plans, 6,654 (93\%) were approved, 144 $(2 \%)$ needed minor modifications, while 351 (5\%) required

\section{Anatomical site distribution (\%)}

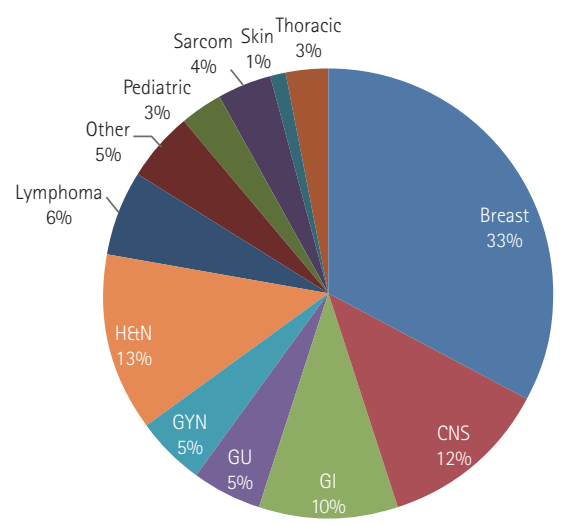

Fig. 1. Anatomical site distribution for presented plans at $Q A$ rounds. CNS, central nervous system; GI, gastrointestinal; GU, genitourinary; GYN, gynecologic; H\&N, head \& neck.

\section{Major modification}

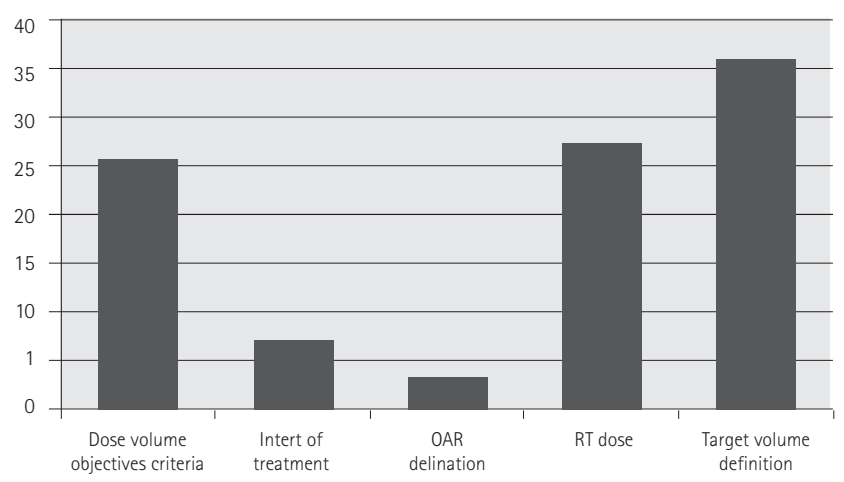

Fig. 2. Percentage distribution of major modification subgroups. $O A R$, organ-at-risk; $R T$, radiation therapy.

major modifications. Major modification included changes in: selected RT dose (96/351, 27\%), target volume definition (127/351, 36\%), OAR delineation (10/351, 3\%), dose volume objectives criteria (90/351,26\%), and intent of treatment (28/351, 8\%) (Fig. 2).

The RT plans which required major modification relative to total plans number for each tumor subtype were as following: head and neck (104/904, 12\%), thoracic (12/199,6\%), gastrointestinal (33/687,5\%), skin (5/106,5\%), genitourinary (16/359, 4\%), breast $(104 / 2387,4 \%)$, central nervous system (36/846, 4\%), sarcoma (11/277, 4\%), pediatric (7/251, 3\%), Iymphoma (10/423, 2\%), gynecological tumors (2/359, 1\%), and other tumor types (11/351, 3\%) (Fig. 2). Among the 351 RT plans which required major modification, 158 (45\%) were re-presented in the $\mathrm{OA}$ rounds after modifications as per $\mathrm{OA}$ round recommendations. 
Table 1. Approved, major and minor modification per each anatomical site

\begin{tabular}{|c|c|c|c|}
\hline & Approved & Minor modification & Major modification \\
\hline Breast & $2,227(93)$ & $56(2)$ & $104(5)$ \\
\hline Central nervous system & 806 (95) & $4(1)$ & $36(4)$ \\
\hline Gastrointestinal & $618(90)$ & $36(5)$ & $33(5)$ \\
\hline Genitourinary & $339(94)$ & $4(1)$ & $16(5)$ \\
\hline Gynecologic & 354 (98) & $3(1)$ & $2(1)$ \\
\hline Head \& neck & $785(87)$ & $15(2)$ & $104(11)$ \\
\hline Lymphoma & $405(96)$ & $8(2)$ & $10(2)$ \\
\hline Other & 333 (95) & $7(2)$ & $11(3)$ \\
\hline Pediatric & $242(96)$ & $2(1)$ & $7(3)$ \\
\hline Sarcoma & 263 (95) & $3(1)$ & $11(4)$ \\
\hline Skin & $96(90)$ & $5(5)$ & $5(5)$ \\
\hline Thoracic & $186(93)$ & $1(1)$ & $12(6)$ \\
\hline
\end{tabular}

Values are presented as number (\%).

Table 2. Approved, major and minor modification per each year

\begin{tabular}{cccc}
\hline Year & Approved & Minor modification & Major modification \\
\hline 2007 & $714(94)$ & $21(3)$ & $25(3)$ \\
2008 & $516(95)$ & $8(1)$ & $23(4)$ \\
2009 & $791(94)$ & $21(3)$ & $29(3)$ \\
2010 & $950(90)$ & $41(4)$ & $68(6)$ \\
2011 & $952(92)$ & $26(3)$ & $62(5)$ \\
2012 & $870(94)$ & $4(1)$ & $48(5)$ \\
2013 & $774(94)$ & $13(2)$ & $41(5)$ \\
2014 & $698(94)$ & $4(1)$ & $33(4)$ \\
2015 & $389(94)$ & & $22(5)$ \\
\hline
\end{tabular}

Values are presented as number (\%).

Table 3. Radiotherapy planning technique for each site

\begin{tabular}{lccc}
\hline & $2 D$ & $3 D-C R T$ & IMRT \\
\hline Breast & - & $2,355^{\text {a) }}(99)$ & $32(1)$ \\
Central nervous system & $8(1)$ & $709(84)$ & $92(13)$ \\
Gastrointestinal & $544(79)$ & $95(27)$ & $51(8)$ \\
Genitourinary & $137(38)$ & $28(8)$ & $127(35)$ \\
Gynecologic & $324(90)$ & $570(63)$ & $7(2)$ \\
Head \& neck & - & $33(8)$ & $334(37)$ \\
Lymphoma & $369(87)$ & $145(41)$ & $21(5)$ \\
Other & $181(52)$ & $175(70)$ & $25(7)$ \\
Pediatric & $56(22)$ & $179(65)$ & $20(8)$ \\
Sarcoma & $67(24)$ & $94(89)$ & $31(11)$ \\
Skin & - & $166(83)$ & $12(11)$ \\
Thoracic & $21(11)$ & & $12(6)$ \\
\hline
\end{tabular}

Values are presented as number (\%).

3D-CRT, three-dimensional conformal radiotherapy; IMRT, intensity-modulated radiotherapy.

${ }^{\text {a) }}$ Forward IMRT. 


\section{Discussion and Conclusion}

Out of the 7,149 RT plans, our study showed that on average $7 \%$ needed modifications. This percentage is close to the 9\% that is reported by Mackenzie et al. [12]; however, the definition for modification in this report required RT plan change before beginning of treatment or before the next fraction. Of those requiring modification, 71\% needed major modification prior to radiation treatment, which means that the patient's treatment has to be delayed until the required changes are implemented and approved.

More than one-third (36\%) of the major modifications were related to PTV which took most of the resources for replanning to assure that the treatment is not delayed much further (Fig. 2). In fact, this is directly related to the CTV delineation (rather than PTV), given that our department follows a standardized PTV margin expansion (beyond the CTV) according to the anatomical tumor subsite, and this took most of the resources for re-planning to assure that the treatment is not delayed much further. The next major changes were in regards to the prescribed RT dose and the plan acceptance. These two items are not automatically classified as major modifications requiring a re-planning. Changing the total RT dose by adding more fractions can easily be done by scaling the prescription in the plan, which can be done during the $\mathrm{QA}$ review rounds. If doing so doesn't exceed the tolerance of the OAR then this can proceed to treatment after recording and approving the new plan data. However, if the dose scaling results in an unacceptable dosimetric values or the regular fraction is replaced by hypofractionation then this become a major modification requiring re-planning.

It is noted that since the introduction of IMRT in 2010 for prostate radiotherapy plans followed by other sites the year after, the number of modifications have increased considerably from the prior 2 years, and then it decreased over the next 2 years. This behaviour is expected after the introduction of any new treatment modality. The $\mathrm{QA}$ team was building experience and then reached a consensus on guidelines for delineation and planning in regards to the IMRT plans. This resulted in subsequently more cases being approved without changes.

Furthermore, our definition of minor modification was close to the definition used in the Canadian QA round reviews $[11,12]$, which defined RT plans as satisfactory, but where issues were raised to consider for future. We recommend minor modification to take place whenever possible even if it has minimal impact on the final outcome, due to laxity in definition of safe practice in radiotherapy in general [14].

In terms of anatomical sites, head \&t neck tumor category exhibited highest percentage of major modifications this is probably related to the higher use of IMRT cases for this site and the anatomical complexity associated with the RT plans. IMRT is known to be associated with more incidental reports [15]. A second contender is breast planning and two main issues contribute to the changes, including nodal irradiation, dose to the heart (for left sided breast cancer patients). All other sites have comparably low percentage of changes.

As part of this study we have recorded that not all modified plans are represented again in the rounds. Out of 351 radiotherapy plans that needed major modifications only 158 (45\%) were represented. However, as per our departmental policy, the chief RTT should ensure that the documented corrections are followed in the edited plan and that the radiation oncologist has signed it. Our charts were changed from paper form into paperless form in 2014. Therefore, the treating physician had to sign the plan and patient chart in paper form prior 2014 and electronic signature thereafter.

The reduction in major modification through the years was not dramatic. Perhaps this is related to the integration of more sophisticated planning technique through the years with more sites as well. It is unrealistic to expect zero errors or modifications in the clinical work of a busy radiotherapy department. In addition, during these years there were physicians and physicist turn over, fresh graduated hired staff. These modifications reflect sharing the experience between physicians and harmonizing the practice.

While we fully support every department to follow the QA rounds assessment, to ensure delivery of high quality RT, we also understand that this would require departmental resources which may not available in low-income countries where treating large number of cancer patients is required and available infra-structures and resources are not sufficient. Perhaps, collaboration with tertiary cancer centers in more developed countries may facilitate the establishment of such approach and enhance the idea of real global cancer management. The future projection of the patient load indicates that it is still manageable to review all radical cases in the $\mathrm{QA}$ rounds, however, we are proactively looking into site specific rounds. This will reduce the load of the staff greatly and focus the effort at the anatomical sites of higher rates of modifications. Site-specific chart rounds have been discussed by others for lung [16], breast [17], and lymphoma [13]. Our aim is to try to reduce the baseline number of cases that require major modification by gaining more experience 
through our $\mathrm{QA}$ rounds in CTV delineation, planning and plan approval prior $\mathrm{QA}$ rounds. For the past 2 years, dedicated educational sessions for radiotherapy plan approval are given to senior residents by our staff. Another initiative at our department aiming to decrease major medications was the quality improvement committee which started by the end of 2015 responsible for standardizing radiotherapy dose and volume for each site and implementing a library for the terms used in target and OAR delineation.

The $Q A$ rounds have shown noteworthy benefits in sharing experience between radiation oncologists and providing knowledge for the team involved in patient care and radiotherapy delivery including dosimetrists, physicists, technicians and nurses. Also the rounds harmonize the practice within the same institution through years of practice and provide a vital education tool for radiation oncology residents [10]. In addition the $\mathrm{OA}$ round records offers a data source for departmental research.

Finally, in the era of personalized oncology practice and precision in radiotherapy, $\mathrm{QA}$ rounds provides a case oriented discussion depending on the case related variables to improve quality and ensure safety [18].

In conclusion, we encourage every RT department to assess its treatment management of patient care by performing a similar analysis study to find their own baseline of rejected cases and try to improve. $\mathrm{QA}$ chart rounds in a multidisciplinary team approach in our experience present a corner stone to improve patient safety and standardize treatment planning.

\section{Conflict of Interest}

No potential conflict of interest relevant to this article was reported.

\section{References}

1. Marks $L B$, Light $K L$, Hubbs $J L$, et al. The impact of advanced technologies on treatment deviations in radiation treatment delivery. Int J Radiat Oncol Biol Phys 2007;69:1579-86.

2. Jaradat I, Mula-Hussain L, Wadi-Ramahi S, et al. Practical steps for establishing ocular plaque therapy in developing countries. Brachytherapy 2012;11:230-6.

3. Khader J, Al-Mousa A, Hijla FA, et al. Requirements and implementation of a lung SBRT program in a developing country: benefits of international cooperation. Int J Radiat Oncol Biol Phys 2016;95:1236-8.

4. Salem A, Al-Rashdan A, Jaradat I, Hashem SA, Almousa A.
Intensity-modulated radiotherapy in head and neck cancer: how safe is safe? Hematol Oncol Stem Cell Ther 2011;4:192.

5. World Health Organization. Radiotherapy risk profile technical manual. Geneva, Switzerland: World Health Organization; 2008.

6. Taghavi Bayat B, Gill S, Siva S, Tai KH, Joon ML, Foroudi F. Tenyear results of quality assurance in radiotherapy chart round. BMC Health Serv Res 2013;13:148.

7. Roques TW. Patient selection and radiotherapy volume definition: can we improve the weakest links in the treatment chain? Clin Oncol (R Coll Radiol) 2014;26:353-5.

8. Purdy JA. Current ICRU definitions of volumes: limitations and future directions. Semin Radiat Oncol 2004;14:27-40.

9. Matuszak MM, Hadley SW, Feng $M$, et al. Enhancing safety and quality through preplanning peer review for patients undergoing stereotactic body radiation therapy. Pract Radiat Oncol 2016;6:e39-46.

10. Lawrence $Y R$, Whiton MA, Symon $Z$, et al. Quality assurance peer review chart rounds in 2011: a survey of academic institutions in the United States. Int J Radiat Oncol Biol Phys 2012;84:590-5.

11. Lefresne $S$, Olivotto IA, Joe $H$, Blood PA, Olson RA. Impact of quality assurance rounds in a Canadian radiation therapy department. Int J Radiat Oncol Biol Phys 2013;85:e117-21.

12. Mackenzie J, Graham G, Olivotto IA. Peer review of radiotherapy planning: quantifying outcomes and a proposal for prospective data collection. Clin Oncol (R Coll Radiol) 2016;28:e192-e198.

13. Shikama N, Oguchi M, Isobe K, et al. Quality assurance of radiotherapy in a clinical trial for lymphoma: individual case review. Anticancer Res 2007;27(4C):2621-5.

14. Ford EC, Terezakis S. How safe is safe? Risk in radiotherapy. Int J Radiat Oncol Biol Phys 2010;78:321-2.

15. Elnahal SM, Blackford A, Smith K, et al. Identifying predictive factors for incident reports in patients receiving radiation therapy. Int J Radiat Oncol Biol Phys 2016;94:993-9.

16. Rooney KP, McAleese J, Crockett $C$, et al. The impact of colleague peer review on the radiotherapy treatment planning process in the radical treatment of lung cancer. Clin Oncol ( $R$ Coll Radiol) 2015;27:514-8.

17. Lymberiou T, Galuszka $S$, Lee $G$, et al. Predictors of breast radiotherapy plan modifications: quality assurance rounds in a large cancer centre. Radiother Oncol 2015;114:17-21.

18. Marks LB, Adams RD, Pawlicki T, et al. Enhancing the role of case-oriented peer review to improve quality and safety in radiation oncology: executive summary. Pract Radiat Oncol 2013;3:149-56. 